\title{
Recent advances in multiuser MIMO systems
}

\author{
Rodrigo C de Lamare ${ }^{1 *}$, Martin Haardt ${ }^{2}$, Michael Joham ${ }^{3}$, Didier Le Ruyet ${ }^{4}$ and David J Love ${ }^{5}$
}

We would like to welcome you to the Special Issue on Recent Advances in Multiuser MIMO Systems of the EURASIP Journal on Wireless Communications and Networking (JWCN). This issue has collected several research results on various aspects of multiuser MIMO systems including resource allocation, precoding and detection algorithms, limited feedback techniques and network MIMO.

MIMO is a key technology for future wireless communication systems. By exploiting the multi-dimensional properties of wireless channels created by multiple transmit and receive antennas, MIMO systems can significantly increase the channel capacity and link robustness of wireless communication, and have been widely adopted in many future wireless communication standards (e.g. WiMAX, 3GPP LTE, etc.). Multiuser MIMO algorithms are recently being considered to enhance wireless communications systems when the number of users, or connections, are greater than one.

Multiuser MIMO systems can be broadly categorized into MIMO broadcast channels and MIMO multiple access channels for downlink and uplink situations, respectively. The achievable gains of multiuser MIMO in wireless systems are yet to be demonstrated in practice and this is currently the subject of intense research efforts. Nevertheless, these techniques are forecast to play a major role in the increase of spectral efficiency of future wireless networks. A number of problems need to be addressed before multiuser MIMO becomes widely adopted. Among these problems are interference mitigation and management, resource allocation, the amount of feedback information for precoding and coordination, implementation and energy consumption issues, scalability and coordination between access points (APs) strategies. The idea of this special issue was to capture recent research contributions and advances in multiuser MIMO systems and their applications to wireless networks. We have received about 60 paper submissions for this Special Issue by the deadline in October 2010.

\footnotetext{
* Correspondence: rcd1500@york.ac.uk

'Department of Electronics, University of York, York, UK

Full list of author information is available at the end of the article
}

After extensive and careful reviews, we accepted 19 high-quality papers that best fit with the topic of multiuser MIMO systems.

We have organized the papers into several groups dealing with multiuser MIMO systems. The first two papers by Duplicy et al. and Papathanassiou et al. focus on multiuser MIMO concepts for long-term evolution (LTE) and for the downlink of IEEE 802.16 m networks, respectively. These papers provide an excellent overview of the role of multiuser MIMO in modern wireless systems.

The second group has two papers that deal with capacity studies. The first paper by Chen et al. investigates the achievable rates of MIMO broadcast channels with feedback-link capacity constraints, whereas the second paper by Shakir et al. examines a bound on the joint decoding capacity of Wyner Gaussian cellular MAC.

The third group of papers focuses on resource allocation strategies for multiuser MIMO systems, which includes techniques for power allocation, user selection and scheduling. The first paper by Brah et al. introduces the Lambert W-function for constrained resource allocation, whereas the second paper by Silva et al. investigates power allocation strategies in multicell systems with precoding. The third paper by Celcer et al. proposes an adaptive utility-based scheduling algorithm for the multiuser MIMO uplink, and the fourth paper by Salim et al. compares various user selection algorithms for achieving SINR targets with average minimum transmit power in multiuser MIMO systems.

Another group of selected papers for this issue specializes in precoding and limited feedback techniques. The first paper by Kaviani et al. investigates optimal multiuser zero-forcing algorithms with per-antenna power constraints for network MIMO. The second paper by Zhang et al. examines vector perturbation algorithms for distributed precoding with limited feedback for CoMP systems. The third paper by Matsumura et al. examines orthogonal beamforming using Gram-Schmidt orthogonalization for multiuser MIMO downlink schemes, whereas the fourth paper by Elliot et al. investigates improved sum-rate maximization for successive zero- 
forcing in multiuser MIMO systems. The fifth paper by Zeydan et al. examines joint iterative beamforming and power adaptation for MIMO ad hoc networks, whereas the sixth paper by Mroueh et al. investigates reduced feedback for selective fading MIMO broadcast channels.

The last group of selected papers deals with aspects of receiver design, space-time coding and reconfiguration. The first paper by An et al. investigates a low-complexity lattice reduction scheme for space-time block-coded (STBC) two-user uplink MIMO systems, whereas the second paper by Medina et al. examines frequencydomain equalization for STBC CDMA systems. The third paper by Ghaffar et al. proposes an interferenceaware receiver structure for multiuser MIMO and LTE. The fourth paper by Kountouriotis et al. studies reconfigurable antennas and configuration selection methods for MIMO ad hoc networks, while the fifth paper by Dong et al. presents a study of adaptive antenna selection and transmit/receive beamforming for large-scale MIMO systems in $60 \mathrm{GHz}$ channels.

We would like to thank all the authors who have submitted their papers to this special issue. We would also like to thank all the reviewers for their time and efforts. Their careful reviews and valuable comments helped us select the papers as well as improve the quality of this special issue. Finally, we hope that the contents of this special issue will serve as good references for your research and that you will enjoy reading the carefully selected papers on the exciting research area of multiuser MIMO systems.

\footnotetext{
Author details

${ }^{1}$ Department of Electronics, University of York, York, UK ${ }^{2}$ Communications Research Laboratory, IImenau University of Technology, IImenau, Germany ${ }^{3}$ Associate Institute for Signal Processing, TU Munich, Munich, Germany ${ }^{4}$ Conservatoire National des Arts et Métiers, CNAM, Paris, France ${ }^{5}$ School of Electrical and Computer Engineering, Purdue University, Indianapolis, IN, USA
}

Received: 28 August 2011 Accepted: 3 November 2011

Published: 3 November 2011

doi:10.1186/1687-1499-2011-157

Cite this article as: de Lamare et al:: Recent advances in multiuser MIMO systems. EURASIP Journal on Wireless Communications and Networking 2011 2011:157.

\section{Submit your manuscript to a SpringerOpen ${ }^{\mathcal{O}}$ journal and benefit from:}

- Convenient online submission

- Rigorous peer review

- Immediate publication on acceptance

- Open access: articles freely available online

- High visibility within the field

- Retaining the copyright to your article

Submit your next manuscript at $>$ springeropen.com 